\title{
BMJ Open Observational retrospective study of US national utilisation patterns and live birth rates for various ovarian stimulation protocols for in vitro fertilisation
}

Vitaly A Kushnir, ${ }^{1,2}$ Sarah K Darmon, ${ }^{1}$ David H Barad, ${ }^{1,3}$ Norbert Gleicher ${ }^{1,3,4,5}$

To cite: Kushnir VA, Darmon SK, Barad DH, et al. Observational retrospective study of US national utilisation patterns and live birth rates for various ovarian stimulation protocols for in vitro fertilisation. BMJ Open 2018;8:e023124. doi:10.1136/ bmjopen-2018-023124

- Prepublication history for this paper is available online. To view these files, please visit the journal online (http://dx.doi. org/10.1136/bmjopen-2017023124).

Received 23 March 2018 Revised 14 June 2018 Accepted 8 October 2018

\section{Check for updates}

(c) Author(s) (or their employer(s)) 2018. Re-use permitted under CC BY-NC. No commercial re-use. See rights and permissions. Published by BMJ.

${ }^{1}$ Center for Human Reproduction, New York, New York, USA

${ }^{2}$ Department of Obstetrics and Gynecology, Wake Forest School of Medicine, Winston-Salem, North Carolina, USA

${ }^{3}$ Foundation for Reproductive Medicine, New York, New York, USA

${ }^{4}$ Stem Cell Biology and

Molecular Embryology Laboratory, The Rockefeller University, New York, New York, USA

${ }^{5}$ Department of Obstetrics and Gynecology, Medical University of Vienna, Vienna, Austria

Correspondence to Dr Vitaly A Kushnir; vkushnir@thechr.com

\section{ABSTRACT}

Objective Alternative ovarian stimulation protocols for in vitro fertilisation (IVF) have grown in popularity. Yet, patient populations best suited for these protocols have not been defined. Our objective was, therefore, to determine national IVF utilisation patterns and live birth rates of various ovarian stimulation protocols.

Design Retrospective cohort study.

Setting Academic-affiliated private fertility centre. Participants Aggregate data published by Society for Assisted Reproductive Technology for autologous IVF cycles performed in the USA during 2014 and 2015 were analysed. IVF cycles were stratified based on ovarian stimulation protocol: 205705 conventional stimulations, 4397 minimal stimulations, 2785 natural cycles and 514 in vitro maturation (IVM) cycles. Repeat cycles could not be determined in this analysis.

Outcome measures Utilisation patterns and age-specific live birth rates for various ovarian stimulation protocols.

Results With advancing female age, utilisation of conventional stimulation protocols decreased, while minimal stimulation and natural cycle IVF increased. Diminished ovarian reserve diagnoses were in all age groups less prevalent in patients undergoing conventional stimulation than with all other protocols. Live birth rates were highest with conventional stimulation at $42.4 \%$, $33.1 \%, 22.1 \%, 11.7 \%$ and $3.9 \%$ for $<35,35-37,38-40$, 41-42 and $>42$ female age groups, respectively. The difference in live birth rates between conventional stimulation and other protocols widened with advancing age from 1.6 -fold to 3.9 -fold among women $<35$ years of age, reaching 4.4-fold to 6.6 -fold among women $>42$ years of age.

Conclusions In comparison to conventional stimulation IVF-minimal stimulation, natural cycle IVF and IVM protocols offer lower but still acceptable live birth rates among young women. These alternative protocols are frequently used in older women and those with diminished ovarian reserve, despite their lower live birth rates. The reasons for this apparent incongruity warrant further careful exploration.

\section{INTRODUCTION}

Selection of ovarian stimulation protocols for in vitro fertilisation (IVF) greatly affects chances of live birth. Multiple studies have
Strengths and limitations of this study

- Retrospective cohort study of aggregate US national data on autologous in vitro fertilisation cycles performed during 2014 and 2015.

- Data were analysed to determine utilisation patterns and age-specific live birth rates for various ovarian stimulation protocols.

- Limitations stem from a lack of standardised definitions and confounding patient characteristics which could not be fully adjusted for.

demonstrated that live birth rates increase in parallel with oocyte yields and number of available embryos for transfer. ${ }^{1-6}$ Yet, ovarian stimulation protocols that, a priori, produce lower oocyte and embryo yields in IVF cycles, have become increasingly popular, ${ }^{7}$ including natural cycle $\mathrm{IVF}^{8}$ minimal stimulation $\mathrm{IVF}^{9}$ and in vitro maturation (IVM). ${ }^{1011}$

Utilisation of these protocols has increased with different motivations. For example, minimal stimulation IVF has been promoted as being more physiological, gentle, patientfriendly and cost-effective, causing controversy. ${ }^{12-14}$ Though cumulative live birth rates with minimal stimulation IVF in a recent randomised controlled trial report were lower than with conventional stimulation, ${ }^{15}$ the same authors, nevertheless, concluded that minimal stimulation IVF for many patients represents an overall superior approach. ${ }^{16}$

Controversy regarding the efficacy of minimal stimulation protocols is further highlighted by two recent review articles which reached quite different conclusions. The first review concluded that in routine practice conventional stimulation is superior to minimal stimulation IVF based of four fundamental issues: prevalence of severe ovarian hyperstimulation syndrome, oocyte/embryo quality, pregnancy/live birth rates and cost. ${ }^{17}$ On the other hand, another review article 


\begin{tabular}{|c|c|c|c|c|c|}
\hline & $<35$ & $35-37$ & $38-40$ & $41-42$ & $>42$ \\
\hline Cycle starts & $\begin{array}{l}83637 \\
(98.5 \%)\end{array}$ & $\begin{array}{l}43661 \\
(97.7 \%)\end{array}$ & $\begin{array}{l}41661 \\
(96.0 \%)\end{array}$ & $\begin{array}{l}21809 \\
(93.7 \%)\end{array}$ & $\begin{array}{l}14937 \\
(87.1 \%)\end{array}$ \\
\hline Cancelled cycles & $5.5 \%$ & $8.3 \%$ & $11.0 \%$ & $13.6 \%$ & $16.4 \%$ \\
\hline Retrievals with no embryos suitable for transfer & $4.0 \%$ & $6.8 \%$ & $11.2 \%$ & $17.9 \%$ & $24.8 \%$ \\
\hline Elective single embryo transfer & $37.0 \%$ & $27.1 \%$ & $17.9 \%$ & $11.4 \%$ & $7.3 \%$ \\
\hline DOR & $5.4 \%$ & $11.7 \%$ & $21.2 \%$ & $34.0 \%$ & $44.2 \%$ \\
\hline Cycle starts & 678 & 627 & 1050 & 922 & 1120 \\
\hline & $(0.8 \%)$ & $(1.4 \%)$ & $(2.4 \%)$ & $(4.0 \%)$ & $(6.5 \%)$ \\
\hline Cancelled cycles & $10.0 \%$ & $12.9 \%$ & $11.5 \%$ & $15.9 \%$ & $15.7 \%$ \\
\hline DOR & $14.0 \%$ & $30.3 \%$ & $46.4 \%$ & $64.1 \%$ & $66.8 \%$ \\
\hline Cycle starts & 451 & 314 & 574 & 443 & 1003 \\
\hline & $(0.5 \%)$ & $(0.7 \%)$ & $(1.3 \%)$ & $(1.9 \%)$ & $(5.8 \%)$ \\
\hline Cancelled cycles & $21.1 \%$ & $26.8 \%$ & $22.1 \%$ & $20.8 \%$ & $24.1 \%$ \\
\hline Retrievals with no embryos suitable for transfer & $12.1 \%$ & $20.4 \%$ & $21.3 \%$ & $29.9 \%$ & $31.4 \%$ \\
\hline Mean no of embryos transferred & 1.2 & 1.2 & 1.2 & 1.2 & 1.2 \\
\hline DOR & $14.9 \%$ & $17.3 \%$ & $29.1 \%$ & $41.3 \%$ & $48.6 \%$ \\
\hline Cycle starts & 120 & 82 & 130 & 93 & 89 \\
\hline
\end{tabular}

Cycle number is based on combined final 2014 and preliminary 2015 US national data reported by Society for Assisted Reproductive Technology.

Proportion of patients with DOR diagnosis are significantly different between ovarian stimulation protocols in all age groups $(p<0.0001)$.

Mean number of embryos transferred is based on final 2014 data.

DOR, diminished ovarian reserve; IVF, in vitro fertilisation; IVM, in vitro maturation.

summarising a heterogeneous group of clinical studies reached more favourable conclusions of minimal stimulation IVF suggesting that its use should be increased worldwide. $^{18}$

Since utilisation patterns and live birth rates for various ovarian stimulation protocols have never been compared on a large scale, we here analyse published US national IVF live birth rates based on type of ovarian stimulation. The purpose of this study was not to confirm or reject claims made in the literature in support of any one of these stimulation protocols. For that purpose, readers are referred to recent publications. ${ }^{17-19}$ To facilitate patient counselling, we here instead, simply, wish to report how in the US utilisation patterns and live birth rates differ at varying ages with various ovarian stimulation protocols with reference point cycle start.
As this study will demonstrate, national US outcome data for IVF largely are contradictory to current utilisation patterns of alternative ovarian stimulation protocols.

\section{METHODS}

As reported in the 2014-2015 publicly available data set of the Society for Assisted Reproductive Technology (SART),${ }^{20}$ we compared female age-stratified IVF live birth for various ovarian stimulation protocols, including conventional and minimal stimulations, natural cycles and IVM cycles. IVF cycles were stratified based on ovarian stimulation protocols: 205705 conventional stimulations, 4397 minimal stimulations, 2785 natural cycle and 514 IVM cycles. Since ovarian reserve is a major predictor of response to ovarian stimulation and ultimately chance 
Table 2 In vitro fertilisation (IVF) pregnancy outcome for each ovarian stimulation protocol stratified by female age group

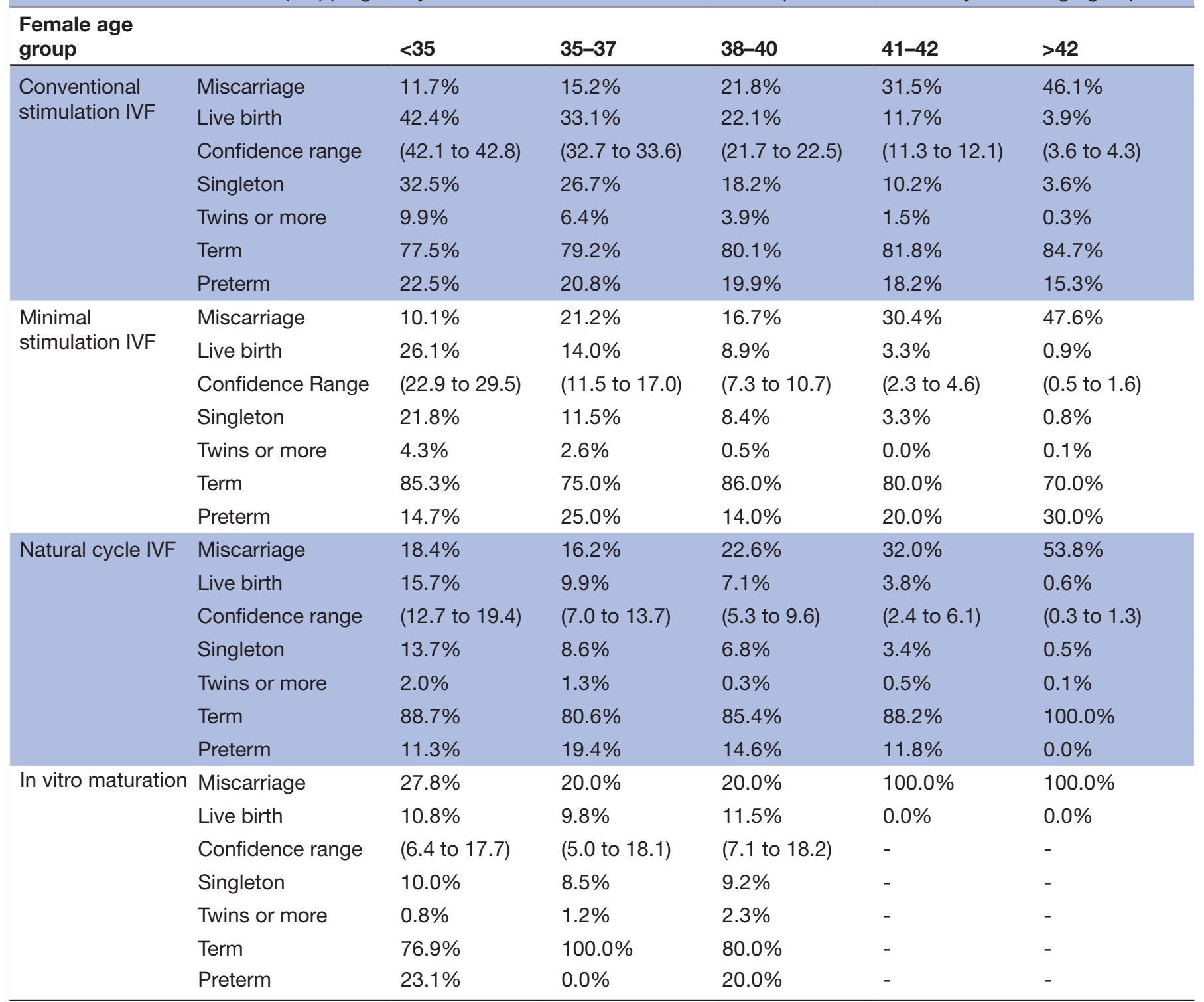

of live birth with IVF, we also performed above analyses specifically for patients with diminished ovarian reserve (DOR) diagnosis for each age group and treatment protocol.

SART reports are based on anonymised aggregate data of US fertility centres, which collectively perform over $90 \%$ of all US IVF cycles. As previously described, these source data undergo annual validation. ${ }^{21}$

SART allows each reporting fertility centre to assign to each IVF cycle the stimulation protocol that is most fitting to the following common definitions: (1) Conventional stimulation, 'administration of injectable gonadotropins for approximately 8-10 days to recruit multiple mature eggs'; (2) Minimal stimulation, 'uses lower doses of injectable gonadotropins than those used for conventional ovarian stimulation. The lower doses of medication may lead to recruitment of fewer eggs than conventional stimulation, the definition ...may vary among clinic as there is no universal standard for minimal stimulation';
(3) Natural cycle, 'requires no fertility medication' and (4) IVM, 'collection of immature eggs that are then incubated in the laboratory prior to IVF'. ${ }^{20}$

Live birth rates are now assessed by SART with reference point cycle start, with first embryo transfers considered, whether fresh or the first frozen-thawed transfer in all-freeze cycles. ${ }^{22}$

Outcome comparisons between stimulation protocols were made using the two-tailed Fisher's exact test, and the Wilson CI for binomial proportions. Conventional stimulation IVF served as the reference for all statistical comparisons. $\mathrm{P}$ values of $<0.05$ were considered statistically significant. All statistical analyses were performed by the centre's principal statistician (SKD), using SAS V.9.4 statistical software.

\section{Patient and public involvement}

Patients were not involved in the design of the study. 


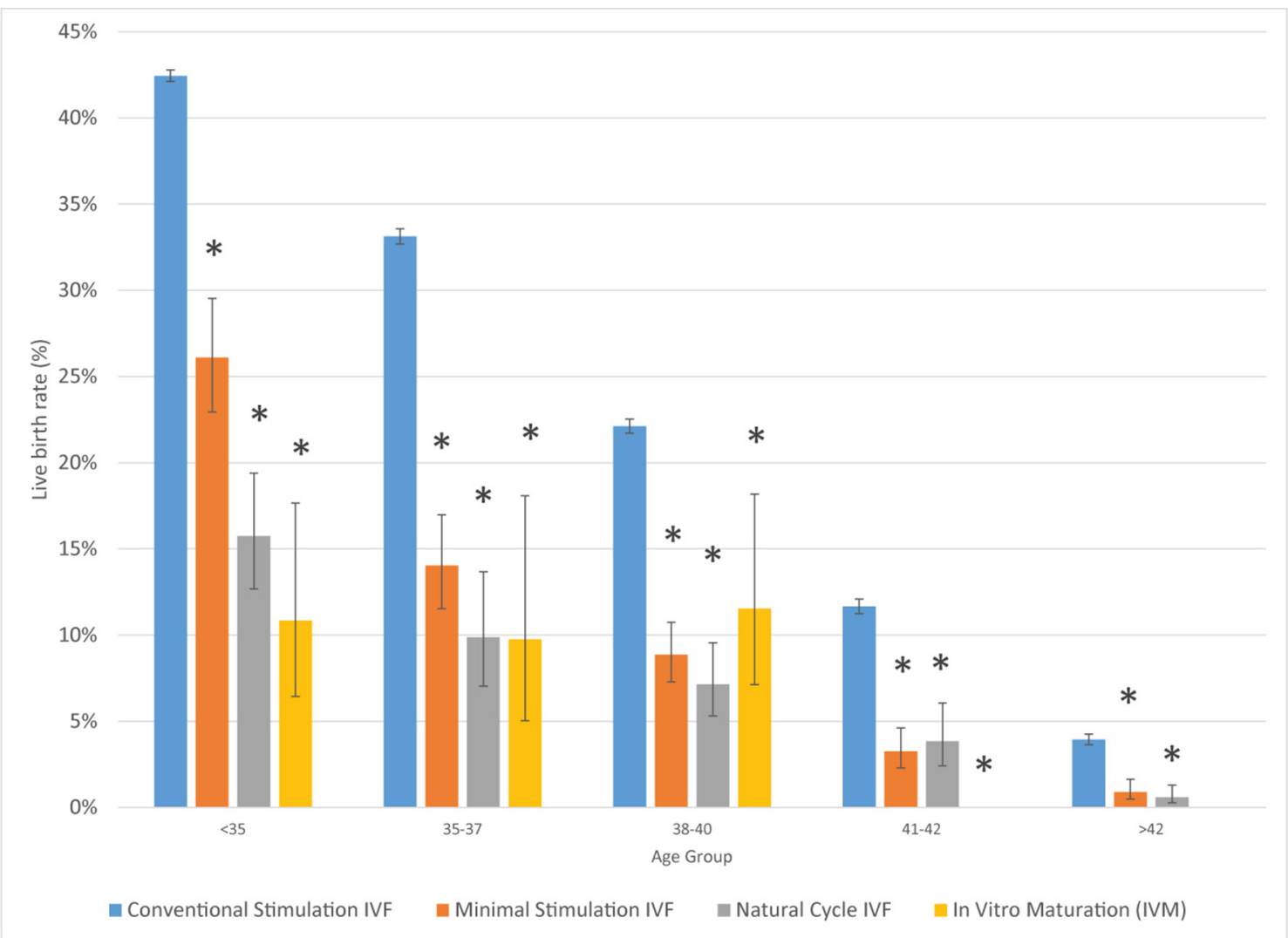

Figure 1 Primary live birth rate per oocyte retrieval cycle for the whole study population stratified by ovarian stimulation protocol. Based on combined final 2014 and preliminary 2015 US national data reported by Society for Assisted Reproductive Technology. Conventional stimulation IVF served as the reference for all statistical comparisons. ${ }^{*} \mathrm{P}<0.05$. IVF, in vitro fertilisation.

\section{RESULTS}

Table 1 summarises the characteristics of IVF cycles for each ovarian stimulation protocol, it is stratified by female age group. Mean numbers of transferred embryos with advancing female age increased more rapidly among women undergoing conventional stimulation IVF than among those undergoing all other stimulation protocols. With advancing age, the number and proportion of conventional stimulation cycles, however, decreased, while minimal stimulation and natural IVF cycles increased. As expected, the proportion of patients with DOR in all groups increased with advancing age but was somewhat lower among women undergoing conventional stimulation IVF than among those undergoing all other stimulation protocols. Interestingly, $57.9 \%$ of all minimal stimulation and natural IVF cycles were performed by only two US IVF centres, suggesting that these two protocols have received only limited acceptance.

Table 2 summarises the pregnancy outcomes for each ovarian stimulation protocol used during the IVF cycle, the data are stratified by female age group. In addition, figure 1 demonstrates in more detail the primary live birth rates for the various ovarian stimulation protocols, stratified by female age. As the figure demonstrates, starting with youngest patients under age 35 years up to oldest patients above age 42 , conventional ovarian stimulations uniformly resulted in the highest live birth rates, followed by minimal stimulations, natural cycles and IVM. While this order was most pronounced in youngest women under age 35 , differences between minimal stimulation, natural cycles and IVM disappeared above age 35 years, though dominance of conventional stimulations over all other stimulation protocols increased with advancing age.

The difference in live birth rates between conventional stimulation and other protocols, thus, widened with advancing female age from 1.6-fold to 3.9-fold among women under 35 years to 4 .4-fold to 6.6 -fold among women above age 42. Excluding data from the above mentioned two centres which performed $57.9 \%$ of all minimal stimulation and natural IVF cycles showed slightly higher live birth rates (between $0.7 \%$ and $6.1 \%$ ) for these protocols in the remaining centres for all age groups, however, the live birth rates remained significantly lower than those achieved with conventional stimulation.

To assess the impact of DOR as a confounder, we separately assessed only patients with DOR (figure 2). As expected, DOR patients across all age groups demonstrated lower live birth rates than the entire study population (figure 1). However, even DOR patients, separately, again demonstrated the widening difference in live birth rates between conventional stimulation and other protocols with advancing female age from 2.8-fold among women under 35 years old to 5.2-fold among women above age 42 . 


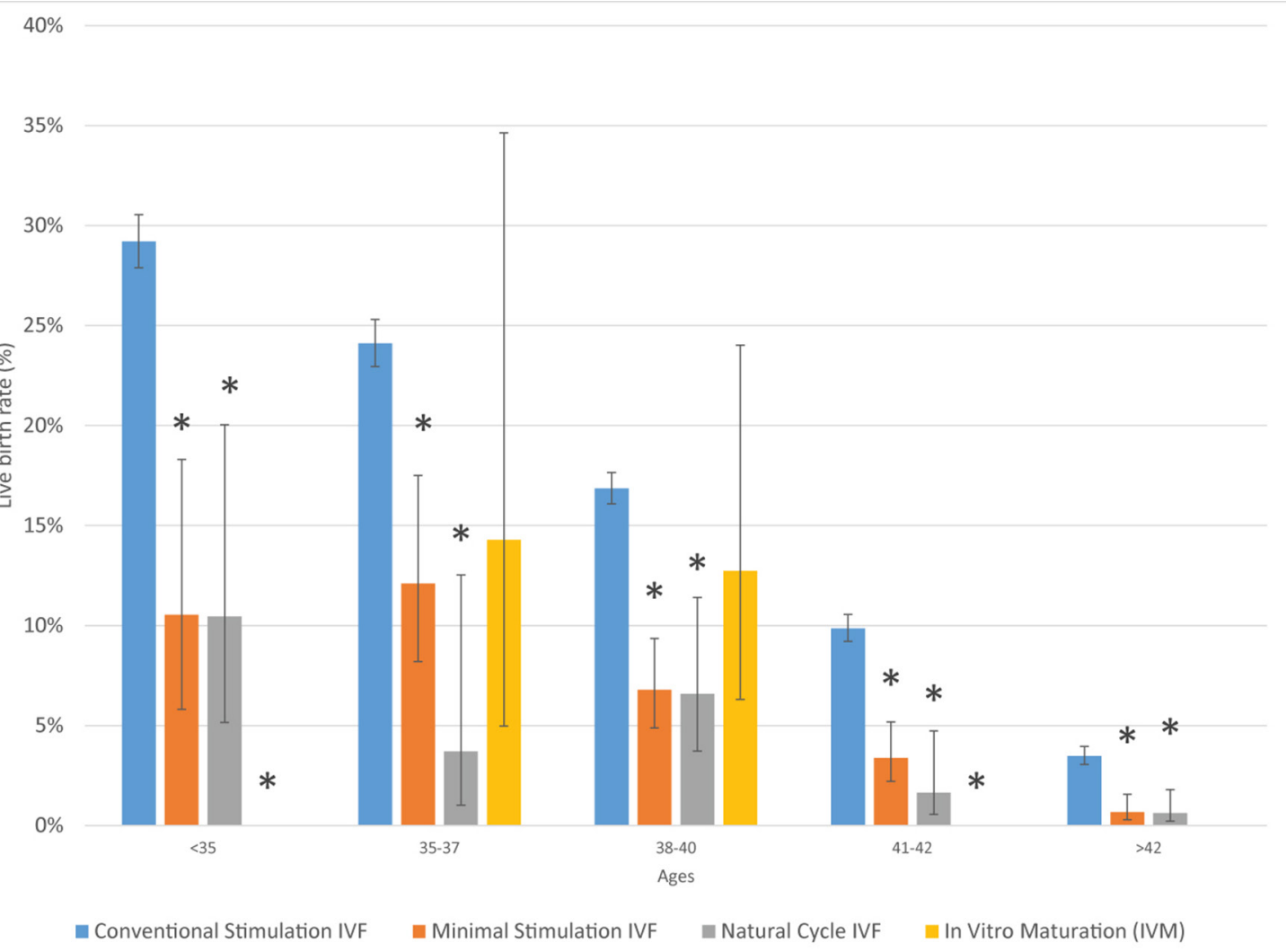

Figure 2 Primary live birth rates per oocyte retrieval cycle for patients with diminished ovarian reserve diagnosis stratified by ovarian stimulation protocol. Based on combined final 2014 and preliminary 2015 US national data reported by Society for Assisted Reproductive Technology. Conventional stimulation IVF served as the reference for all statistical comparisons. ${ }^{*} \mathrm{P}<0.05$. IVF, in vitro fertilisation.

\section{DISCUSSION}

As expected, here presented data confirm universally declining live birth rates with advancing female age. However, somewhat unexpected, the data also reveal contradictory findings to current practice patterns. For example, as table 1 demonstrates, alternative stimulations to conventional stimulations are increasingly used with advancing female age; yet, as figure 1 demonstrates, especially minimal stimulation and natural cycle IVF, while still producing lower live birth rates than conventional stimulation, are clearly more effective in younger women under age 35 than at older ages.

Especially minimal ovarian stimulation with a $26.1 \%$ live birth rate and natural cycle IVF with a $15.7 \%$ live birth rate in young women may be considered potential alternatives to conventional stimulation, even though conventional IVF at $42.4 \%$ clearly produced higher live birth rates. Here observed live birth rates for minimal stimulation and natural cycles in women under age 35 are, indeed, surprisingly robust.

Though the DOR diagnosis was somewhat more common among patients undergoing alternative than conventional stimulations, this difference in DOR prevalence, at most, only partially explains the large difference in live birth rates (figure 1) since restricting the analysis to only patients with DOR did not substantially alter the findings (figure 2). We were not able to analyse other infertility diagnosis such as polycystic ovary syndrome (PCOS) in the present study. It will be important to follow up this analysis for other infertility diagnoses, it is especially interesting to study efficacy of IVM protocols in patients with PCOS. Such an approach may help to identify patients who are best candidates for various protocols.

Our study is particularly timely since it shows that national outcome data from routine clinical practice, contradicts observations from small clinical trials, which have recently been used to promote increased worldwide utilisation of minimal stimulation IVF. $^{18}$

We previously noted that, after female age, number of oocytes retrieved and embryos available for transfer are the most important predictors of live births in IVF cycles. ${ }^{53-25}$ Since implantation rates decline and aneuploidy rates increase with advancing female age, the importance of oocyte and embryo numbers increases because more embryos can be safely transferred into the uterus to compensate for lower implantation rates. Younger women with high implantation rates, in contrast, will often, even with only few embryos, still conceive.

Here presented findings, therefore, make clinical sense but are not reflected in how these alternative stimulations are currently clinically used in the USA. Cumulative live birth rates (per embryo cohort in a single cycle) would, likely, favour conventional stimulation even more profoundly, since these protocols are more likely to result 
in surplus transferable embryos than any of the alternative protocols.

This analysis is limited by lack of a standardised definition of minimal stimulation IVF; SART permits individual fertility centres to designate the most fitting stimulation type for each cycle. Additionally, because this analysis is based on aggregate data, we were except for age and diagnosis of DOR, not able to assess confounding patient characteristics, including number of prior IVF attempts and repeat cycles. We, therefore, cannot rule out undiscovered patient selection biases for individual stimulation protocols. It is possible that some patients undergoing stimulation with alternative protocols had prior conventional stimulation with very low oocyte and embryo yields. Moreover, a retrospective study design does not permit to control for various factors which led to the selection of a stimulation protocol by a physician for each patient. We also note that there is an imbalance in the size of the study groups in our analysis with more than $90 \%$ of cycles in all age groups receiving conventional stimulation. However, it is important to note that by analysing aggregate national data for the entire population of patients rather than a sample of the population the risk of selection bias is somewhat mitigated. Despite above noted limitations, it is unlikely that adjustments for such biases would substantially change the principal findings given the large sample size and that live birth rates were 1.6-fold to 6.6-fold higher with conventional stimulation than all other protocols. We also note that most minimal stimulation and natural IVF cycles were performed by only two fertility centres, where selection of these protocols is likely more a reflection of practice patterns than biased patient selection of poor prognosis patients. Indeed, while these two centres reported marginally lower live birth rates than other centres, excluding their data from the analysis did not substantially alter the principal findings.

Here presented data are, in addition, also consistent with other reports: Silber et al, for example, recently reported in a large number of natural cycles that the chance of live birth per oocyte was 26\% under age 35 but only $1 \%$ above age 42 years. ${ }^{8}$ González-Foruria et al also concluded that natural cycle IVF should be restricted to younger women under age $35,{ }^{26}$ while Check et al reported similar outcomes for conventional and minimal stimulation cycles under age 35 years but clearly superior outcomes for conventional stimulation at older ages. ${ }^{27}$

The literature also supports our observation that conventional ovarian stimulation at all ages, including in women under age 36 , produces higher live birth rates than here investigated alternative stimulations. A recent randomised controlled trial over a 6-month period demonstrated clearly lower cumulative live birth rates with minimal stimulation IVF than conventional stimulation. ${ }^{15}$ When paired with strict single embryo transfer policy, a recent European analysis of cost-effectiveness found that three to six minimal stimulation cycles were comparable in cost to one conventional stimulation cycle. ${ }^{28}$ This observation is relevant regarding the recently reported observation that the wide acceptance by Japanese IVF centres of minimal ovarian stimulation (with blastocyst stage elective single embryo transfer) has resulted in a loss of two-thirds of the national fresh IVF cycle live birth rate over the last decade, while concomitantly tripling the number of IVF cycle starts. ${ }^{19}$

\section{CONCLUSIONS}

Because live birth rates are significantly lower with minimal stimulation IVF than conventional IVF in this analysis of national data, cautious use of minimal stimulation protocols in carefully selected patients rather than their universal application is warranted. Alternative stimulation protocols including minimal stimulation, natural cycle IVF and IVM appear relatively ineffective in women older than 40 and younger women with DOR. Despite these observations, such protocols maybe still be useful in patients with severe DOR who previously did not respond to conventional stimulation.

Acknowledgements The authors wish to thank all SART members for providing clinical information to the SART database for use by patients and researchers. Without the efforts of SART members, this research would not have been possible.

Contributors VAK, DHB and NG developed the concept of the study. All authors contributed to data accumulation. SKD and VAK contributed to data analysis. All authors contributed to data interpretation. VAK wrote the manuscript. All authors contributed to revisions of the manuscript and approved of the final submission. VAK took responsibility for the accuracy of the data analysis.

Funding This study was funded by intramural funds from the Center for Human Reproduction and by grants from The Foundation for Reproductive Medicine.

Competing interests VAK previously served as a consultant to the CDC. The Center for Human Reproduction (CHR) annually routinely reports IVF outcome data to CDC and SART. NG, DHB and VAK are listed as co-owners of several already awarded and still pending US patents, none related to the topic of this manuscript. NG is a shareholder in Fertility Nutraceuticals, LLC and owner of the CHR. NG and DHB receive patent royalties from Fertility Nutraceuticals, LLC. NG and DHB have received research support, travel funding and lecture fees from various Pharma and medical device companies, none, in any way related to this manuscript.

Patient consent Not required.

Ethics approval Because this study investigated only publicly available anonymised data, it received expedited IRB approval.

Provenance and peer review Not commissioned; externally peer reviewed.

Data sharing statement Source data are available from the Society for Assisted Reproductive Technology: https://www.sartcorsonline.com/rptCSR_PublicMultYear. aspx?reportingYear $=2015$ or by contacting the corresponding author.

Open access This is an open access article distributed in accordance with the Creative Commons Attribution Non Commercial (CC BY-NC 4.0) license, which permits others to distribute, remix, adapt, build upon this work non-commercially, and license their derivative works on different terms, provided the original work is properly cited, appropriate credit is given, any changes made indicated, and the use is non-commercial. See: http://creativecommons.org/licenses/by-nc/4.0/.

\section{REFERENCES}

1. Baker VL, Brown MB, Luke B, et al. Association of number of retrieved oocytes with live birth rate and birth weight: an analysis of 231,815 cycles of in vitro fertilization. Fertil Steril 2015;103:931-8.

2. Drakopoulos P, Blockeel C, Stoop D, et al. Conventional ovarian stimulation and single embryo transfer for IVF/ICSI. How many oocytes do we need to maximize cumulative live birth rates after utilization of all fresh and frozen embryos? Hum Reprod 2016;31:dev316-76. 
3. Vaughan DA, Leung A, Resetkova N, et al. How many oocytes are optimal to achieve multiple live births with one stimulation cycle? The one-and-done approach. Fertil Steril 2017;107:397-404.

4. Ji J, Liu Y, Tong XH, et al. The optimum number of oocytes in IVF treatment: an analysis of 2455 cycles in China. Hum Reprod 2013;28:2728-34.

5. Gleicher N, Kushnir VA, Sen A, et al. Definition by FSH, AMH and embryo numbers of good-, intermediate- and poor-prognosis patients suggests previously unknown IVF outcome-determining factor associated with AMH. J Trans/ Med 2016;14:172.

6. Practice Committee of the American Society for Reproductive Medicine. Electronic address: ASRM@asrm.orgPractice Committee of the Society for Assisted Reproductive Technology. Guidance on the limits to the number of embryos to transfer: a committee opinion. Fertil Steril 2017;107:901-3.

7. Edwards RG. IVF, IVM, natural cycle IVF, minimal stimulation IVF time for a rethink. Reprod Biomed Online 2007;15:106-19.

8. Silber SJ, Kato K, Aoyama N, et al. Intrinsic fertility of human oocytes. Fertil Steril 2017;107:1232-7.

9. Teramoto S, Kato O. Minimal ovarian stimulation with clomiphene citrate: a large-scale retrospective study. Reprod Biomed Online 2007;15:134-48.

10. Dahan $\mathrm{MH}$, Tan SL, Chung J, et al. Clinical definition paper on in vitro maturation of human oocytes. Hum Reprod 2016;31:1383-6.

11. Reinblatt SL, Buckett W. In vitro maturation for patients with polycystic ovary syndrome. Semin Reprod Med 2008;26:121-6.

12. Flisser E, Scott RT, Copperman AB. Patient-friendly IVF: how should it be defined? Fertil Steril 2007;88:547-9.

13. Pennings $\mathrm{G}$, Ombelet $\mathrm{W}$. Coming soon to your clinic: patient-friendly ART. Hum Reprod 2007;22:2075-9.

14. van Empel IW, Nelen WL, Hermens RP, et al. Coming soon to your clinic: high-quality ART. Hum Reprod 2008;23:1242-5.

15. Zhang JJ, Merhi Z, Yang M, et al. Minimal stimulation IVF vs conventional IVF: a randomized controlled trial. Am J Obstet Gynecol 2016;214:96.e1-96.e8

16. Zhang J. Resurgence of minimal stimulation in vitro fertilization with a protocol consisting of gonadotropin releasing hormoneagonist trigger and vitrified-thawed embryo transfer. Int J Fertil Steril 2016;10:148-53.
17. Orvieto R, Vanni VS, Gleicher N. The myths surrounding mild stimulation in vitro fertilization (IVF). Reprod Biol Endocrinol 2017;15:48

18. Nargund G, Datta AK, Fauser B. Mild stimulation for in vitro fertilization. Fertil Steril 2017:108:558-67.

19. Kushnir VA, Barad DH, Albertini DF, et al. Systematic review of worldwide trends in assisted reproductive technology 2004-2013. Reprod Biol Endocrinol 2017;15:6.

20. Society for Assisted Reproductive Technology. National Data Summary. https://www.sartcorsonline.com/rptCSR_PublicMultYear. aspx? reportingYear=2015 (Accessed 10 May 2017).

21. Kushnir VA, Darmon SK, Shapiro AJ, et al. Utilization of third-party in vitro fertilization in the United States. Am J Obstet Gynecol 2017;216:266.e1-266.e10.

22. Doody KJ. Cryopreservation and delayed embryo transfer-assisted reproductive technology registry and reporting implications. Fertil Steril 2014;102:27-31.

23. Gleicher N, Darmon SK, Kushnir VA, et al. How FSH and AMH reflect probabilities of oocyte numbers in poor prognosis patients with smal oocyte yields. Endocrine 2016;54:476-83.

24. Gleicher N, Vega MV, Darmon SK, et al. Live-birth rates in very poor prognosis patients, who are defined as poor responders under the Bologna criteria, with nonelective single embryo, two-embryo, and three or more embryos transferred. Fertil Steril 2015;104:1435-41.

25. Vega MG, Gleicher N, Darmon SK, et al. IVF outcomes in averageand poor-prognosis infertile women according to the number of embryos transferred. Reprod Biomed Online 2016;33:370-5.

26. González-Foruria I, Peñarrubia J, Borràs A, et al. Age, independent from ovarian reserve status, is the main prognostic factor in natura cycle in vitro fertilization. Fertil Steril 2016;106:342-7.

27. Check JH, Choe JK, Cohen R, et al. The effect of conventional vs. mild ovarian hyperstimulation on the total number of live babies born from a given oocyte retrieval. Clin Exp Obstet Gynecol 2015;42:571-2

28. Groen $\mathrm{H}$, Tonch $\mathrm{N}$, Simons $\mathrm{AH}$, et al. Modified natural cycle versus controlled ovarian hyperstimulation IVF: a cost-effectiveness evaluation of three simulated treatment scenarios. Hum Reprod 2013;28:3236-46. 